\title{
Panorama de las prácticas políticas: una aproximación desde cultura participativa y cultura política
}

\author{
Gabriel Corral Velázquez ${ }^{1 *}$, Rosario Barba González²
}

\begin{abstract}
Resumen
La cultura participativa (Jenkins, 2008) es un término que en los últimos años ha permitido explicar el fenómeno de las nuevas interacciones que han ido surgiendo a partir de la relación con nuevas plataformas mediáticas y entre las propias audiencias. La cultura participativa describe cambios particulares en el escenario mediático de forma que las barreras tradicionales se han ido deslavando poco a poco (Barba, 2017). Dentro de estas transformaciones, la relación contemporánea con el ecosistema digital motiva a la divulgación de información de manera más amplia.

En correspondencia, se puede señalar que la participación puede ser comprendida como un ejemplo de cultura política, en tanto que se adquiere el cómo y en qué participar políticamente. Este trabajo pretende abrir un camino reflexivo entre la cultura política, en su abordaje tradicional, y el desdoblamiento de este entendimiento desde el concepto de cultura participativa. Este trabajo, desarrollado en la ciudad de Querétaro, es una exploración a este respecto a partir de un sondeo no probabilístico que empieza a buscar las formas de participación política, previo a la elección de 2018.

\section{Abstract}

Participatory culture (Jenkins, 2008) is a term that in recent years has allowed explaining the phenomenon of new interactions that have emerged from the relationship with new media platforms and between the audiences themselves. Participatory culture describes particular changes in the media scenario so that traditional barriers have gradually been washed away (Barba, 2017). Within these transformations, the contemporary relationship with the digital ecosystem motivates the dissemination of information more widely.

Correspondingly, it can be pointed out that participation can be understood as an example of political culture, as it is acquired how and in what to participate politically. This work aims to open a reflective path between political culture, in its traditional approach, and the unfolding of this understanding from the concept of participatory culture. This work, developed in the city of Querétaro, is an exploration in this regard from a non-probabilistic survey that begins to seek forms of political participation, prior to the 2018 election

\section{Palabras Clave}

Cultura participativa, Cultura política, Querétaro, Elección 2018.

\section{Keywords}

Participatory culture, Political culture, Queretaro, 2018 election.

${ }^{1}$ Doctor en Estudios Científico Sociales (ITESO - Universidad Jesuita de Guadalajara) Profesor en la Universidad Autónoma de Querétaro. ${ }^{2}$ Doctora en Estudios Socioculturales (Universidad Autónoma de Aguascalientes) Profesora en la Universidad Autónoma de Querétaro.

*Autor para correspondencia: corral@uaq.mx

*Autor para correspondencia: rosario.barba@uaq.mx
\end{abstract}

\section{Acercamiento a la cultura participativa y la cultura política}

El concepto cultura participativa ofrece la posibilidad de analizar un tipo de audiencia-usuario, que configura, a partir 
de sus prácticas, una forma de cultura que solo es posible bajo el contexto sociohistórico actual de desarrollos tecnológicos digitales convergentes, generado por las industrias culturales en este periodo de globalización. En ese contexto, las prácticas políticas diversifican sus matices y entornos de manifestación. El interés del trabajo presentado es vincular el análisis de la cultura política, con su trayectoria en trabajos cuantitativos de encuestas y sondeos con una mirada desde la cultura participativa. Una primera aproximación a este cruce se realizó partir de un sondeo llevado a cabo en la ciudad de Querétaro. Los resultados se contrastan con la información que se tiene para el país, permitiendo realizar anotaciones que abran camino en esta dirección de reflexión.

Con los referentes estructurales de la cultura participativa y los de los sujetos se pueden establecer articulaciones en al menos dos sentidos. Por un lado, la referencia a un entorno global que permite una participación más activa de los usuarios en los bienes de consumo mediático, que los involucra y los hace partícipes de la gestión y distribución de estos. Por el otro, los propios referentes de los sujetos que los incluye en grupos de referencia. Lo anterior hace relevante el análisis en virtud de conocer y observar estas comunidades que han permitido formas innovadoras de consumo y producción de significados compartidos, con relación a productos mediáticos y la relación que esta adquiere en su vida cotidiana.

De acuerdo con Mattelart (2003), a partir de la segunda mitad de la década de los noventa y los primero años del siglo XXI, el escenario global y político global se ha visto envuelto en una serie de transformaciones. Mismas que han sido producto de las dinámicas mediáticas digitales y de la emergencia de actores políticos que, su vez, han dado lugar al surgimiento de nuevos movimientos, algunos espontáneos, otros no, y que manifiestan la posibilidad de participar de muy diversa forma en las actividades públicas y públicas mediáticas.

Los cambios se han dado, principalmente, en la esfera de lo público (Habermas, 1981). Esta esfera invita a la discusión de los asuntos públicos y apunta a la cultura política, alimentada por los periódicos que incluyen comentarios políticos y sátiras diversas y que se convirtieron en "el dispositivo de la concurrencia misma", influyente como mediador pero libre aún de ser "un medio para la cultura del consumo" (Habermas, 1981: 128).

En este sentido, resulta relevante conocer la forma en cómo la esfera de lo público ofrece un vínculo para la configuración de la cultura política y la emergencia de la cultura participativa como resultado del contexto actual.

Con el desarrollo masivo de los medios de comunicación, el fenómeno de la publicidad se desvinculó de toda participación en un espacio común (Thompson, 1998). Se desespacializó tanto, que dejó de basarse en diálogo alguno. La interacción mediática cambió para siempre a la polis liberal: la co-presencia necesaria en los espacios públicos fue sustituida por una publicidad que no requería un público físico y un espacio concreto. Nació la simultaneidad desespacializada que se estudia en todas las escuelas de comunicación:

Personas distantes podían ser vistas de manera virtualmente simultánea, podían oírse en el mismo instante en que hablaban o verse en el mismo momento en que actuaban, a pesar de que no compartían el mismo lugar físico con los individuos para los cuales eran visibles. (Thompson, 1993: 283)

Con el nacimiento de la alta visibilidad mediática, lo que en un principio legitimó el acceso de la prensa al mundo secreto del poder, terminó convirtiéndose en un ambiente informativo más intenso, más extenso y menos controlable. En él, las fronteras entre lo privado y lo público se hundieron para siempre y una emergente cultura política, basada en la confianza y el carácter de los líderes, arrinconó la política ideológica que marcó las sociedades durante gran parte de los siglos XIX y XX (Thompson, 1998).

En este cambio cultural, la visibilidad era prerrogativa de los grandes consorcios (Thompson, 1998) pues ellos definían la publicidad mediática, o la capacidad de ser vistos y oídos en un campo de visión donde no existen ya encuentros cotidianos con otros. No se puede hablar de esfera pública sin comprender que hoy en día existen nuevas formas de interacción y visibilidad que han alterado el carácter simbólico de la vida social.

Las prácticas y los discursos adquieren un valor simbólico distinto a partir de la visibilidad o invisibilidad de los sujetos. 
Quién puede y quién no puede participar en los espacios de visibilidad, que son los medios de comunicación, sirve para acotar la naturaleza democrática de la esfera pública. Para ello se precisa una reconfiguración de la propuesta original de Habermas (1981), labor realizada por Thompson (1998) bajo una propuesta de análisis contextual. Finalmente, la configuración de la esfera pública es resultado de las condiciones sociales y políticas en las cuales se debaten los asuntos de interés colectivo.

Desde Thompson (1998), se reconoce a la recepción como un proceso cotidiano habilidoso. Bajo las condiciones de un mundo globalizado y en un entorno de medios convergentes, los individuos desarrollan habilidades particulares de manejo de información. Estas les permiten integrarse en las nuevas estructuras de transmisión de información a través de canales y géneros creativos. Implica una relación con una amplia variedad de medios tecnológicos que permite consumir, archivar, transformar y recircular contenidos mediáticos (Jenkins, 2009).

En ese tenor, la cultura participativa refiere a una nueva forma de relación entre las audiencias y plataformas mediáticas que emerge del entorno de globalización y convergencia mediática. Los patrones de consumo mediático se alteran conforme los usuarios aprenden e integran formas nuevas de interactuar con los contenidos. La interacción entre medios y contenidos proviene desde el principio. Sin embargo, se rastrea desde la fotocopiadora y posteriormente con el VHS y su grabación; cuando los consumidores comenzaron a ganar el control de cuándo y cómo obtener los contenidos, de almacenar información a la que de otra forma no se hubiera podido tener acceso, y a tomar las herramientas que facilitaran la producción mediática no profesional o industrial. Luego, con la red y los procesos de digitalización se abrió un nuevo espacio para la discusión y el intercambio del contenido mediático. A través de Internet se visibilizaron todo tipo de producciones mediáticas (Thorburn et al., 2003).

La cultura participativa, a últimas fechas, dejó de ser invisible y subterránea (Jenkins, 2006). En un entorno diverso, las competencias de codificación y decodificación de información o contenidos simbólicos se diversifican. Las habilidades comprometidas con la utilización de soportes técnicos, permite y requiere que quienes desean insertarse, adquieran recursos para sus procesos de interpretación y apropiación (Thompson, 1998).

En la cultura participativa, el consumo deviene producción, lectura, escritura, expectación y participación. La interactividad de la audiencia no funciona de forma autónoma, sino que opera junto a los poderes de las industrias culturales. Los conglomerados protegen sus intereses comerciales. Paralelamente, las audiencias, buscan mayor poder e independencia. No se trata de tecnologías que vengan a liberar al espectador sino de interacciones entre consumidores y sus medios, entre consumidores y sus textos y entre consumidores y otros consumidores (Barba, 2017).

La cultura participativa toma forma en la intersección entre tres tendencias vinculadas directamente con la convergencia mediática. Por un lado, un desarrollo tecnológico que permite a los usuarios archivar, anotar, apropiar y recircular contenido mediático. Por el otro, la promoción del DIY en la producción mediática configura un discurso de cómo los consumidores aprovechan esas herramientas. Finalmente, las tendencias económicas que favorecen la integración horizontal de los medios y el flujo de ideas y narrativas entre canales, por lo que demandan distintos modos de ser audiencia (Scolari, 2008).

La característica principal de la cultura participativa es el nivel de interacción y compromiso que implica. Un desempeño activo es requerido, tanto para producir como para consumir. Internet tiende a volverse un medio en el que esta actividad es posible y habilitada (Jenkins, 2006). Mientras se crean y comparten contenidos, se pueden generar lazos sociales. Por lo tanto, el modelo de la cultura participativa genera oportunidades para habilidades apreciadas en el mundo no virtual: provee herramientas que potencian el modelo de enseñanza y aprendizaje entre pares, cuestiona la propiedad intelectual, propone una diversidad de expresiones culturales e, incluso, moviliza concepciones de una ciudadanía empoderada (Jenkins, 2009).

Jenkins (2006) observa cuatro categorías de cultura participativa: afiliación, expresión, solución colaborativa de problemas y circulación. Con afiliación refiere a la membresía, formal e informal, a comunidades virtuales. Esto se debe a que la participación en redes sociales es una competencia cultural esencial dentro de la cultura participativa. 
Las expresiones tienen que ver con la producción de nuevas formas creativas. Internet cuenta con las herramientas para confeccionarlas de forma relativamente fácil, así como con plataformas para hacer circular esas confecciones a través de redes sociales. La solución colaborativa de problemas ocurre cuando varios usuarios trabajan juntos hacia un mismo objetivo.

La categoría de circulación se aplica para la disposición del flujo mediático y de información. El ejemplo que menciona el autor para este caso son los blogs y, más recientemente, los vlogs. Su relevancia recae en ser el espacio de creación de contenido, pero también de crítica y opinión acerca de otro contenido, creado por usuarios o por grandes compañías. Es decir, hacen circular producciones, pero también promueven, o no, el flujo.

Es evidente que las cuatro categorías se encuentran estrechamente entretejidas y mutuamente dependientes. Los contenidos, las opiniones y la información circulan por medio de redes sociales y son las mismas que promueven la producción, la opinión y la participación en la solución de problemas. Aparentemente, los usuarios dan forma a un entorno en el que se habilitan para crear y distribuir contenido por fuera de los límites tradicionales impuestos financiera, política o legalmente. Sin embargo, en el estado de cuestión se puede entrever que hay otras partes que se incrementan de la cultura participativa, por ejemplo, el poder de persuasión de lo digital se ve exacerbado. De esta forma, la dependencia en la tecnología aumenta y la información que en ella se genera cobra mayor relevancia en el desarrollo de la cotidianeidad. De esta forma estimula la integración de la conectividad de Internet a distintos escenarios (Larabie, 2011).

Entonces se argumenta que, en este contexto, la ciudadanía puede adquirir más espacios para ejercer su poder. Hipotéticamente, por medio de la cultura participativa se generan nuevos espacios para la discusión de lo público. Pero esto puede ser problemático cuando los usuarios asumen que es un espacio sin control o que se trata de lugares en donde no se ejerce algún tipo de poder. La realidad es que el poder se ejerce. Las grandes corporaciones también buscan la forma de beneficiarse (Barba, 2017).

Uno de estos espacios, que aparece como privilegiado, son las plataformas de redes sociales. Es claro que replican lo positivo como lo negativo. Expertos ven con pesimismo las conversaciones que, en particular sobre política, pueden desarrollarse en este entorno. Como ejemplo se encuentra la breve discusión que sostuvieron John y Kligler-Vilenchik en el blog de Jenkins (Henry Jenkins, 2019). Sin embargo, aun en el trabajo de ambos autores, aparecen las redes como un espacio en el que se expresa la cultura participativa cívica o política.

Para llevar a cabo el vínculo entre la cultura participativa y la cultura política emergente, Thompson (1998) establece que la esfera pública y su evolución estuvo vinculada, inexorablemente, con la prensa periódica; pues esta misma prensa brindó un nuevo foro para el debate público. Con la llegada de los medios de difusión se construyó el marco mediático.

El marco mediático impone un cambio normativo, la sociedad de masas o sociedad de los medios, la cual desborda el ámbito de interacción de la comunicación política y el debate "racional" que se verificaba en un espacio ceñido por el tiempo y el territorio, al de la mediatización de la vida pública, donde los dispositivos son tecnológicos (medios) e institucionales (el estado y sus instituciones), y el público no está limitado al cuerpo de una región o nación. Los públicos modernos, en oposición a las comunidades tradicionales, son conjuntos de personas que, incluso sin estar en interacción persistente, incluso sin forma alguna de presencia mutua, están sometidas a los mismos estímulos sociales (Andrade, 2009: 93).

Los medios de comunicación serían, pues, el núcleo rector del nuevo espacio público pero la venta de información al público no construye una comunidad de ciudadanos críticos, sino que genera una representación mediada de la realidad social. Lo anterior describe una idea intervenida por elementos cognitivos tanto como por condiciones de producción de cada medio. De esta manera se describe cómo el discurso está impregnado de imaginario político, lo que supone aspectos de la realidad y la fantasía de esa comunidad (Andrade, 2009).

En la esfera pública, los actores toman posición en el debate a partir de su acción discursiva, que se manifiesta en prácticas concretas. La acción del discurso no refiere solamente al ejercicio linguístico, sino que se hace patente a partir de prácticas de referencia. Por lo tanto, a través de él, se ponen de manifiesto las relaciones en la esfera pública y el proceso de democratización y prácticas, tanto emergentes 
como arraigadas, de una cultura política en la esfera pública. En este sentido, la cultura política es representada por sistemas de valores, representaciones simbólicas e imaginarios colectivos, que se ponen de manifiesto en los discursos y prácticas observados en la esfera pública (Almond and Verba, 2001).

\section{El contexto político de Querétaro en México}

García (2018), describe una cultura de desafección política en dos dimensiones; con respecto a lo institucional y de falta de compromiso político. México es uno de los países de Latinoamérica con mayor desafección, en cuanto a que existe una sensación generalizada de desconfianza e impotencia con respecto a los procesos políticos.

En el país, desde la Encuesta Nacional sobre Cultura Política y Prácticas Ciudadanas, cuyos últimos resultados son de 2012, y otros recursos (García, 2018), se encontró que la actitud de poco interés en la política. Esto, se relaciona con la percepción de que la política es algo muy complicado y una proporción de cuatro sobre diez se piensan con pocas posibilidades de participar en ella y sus instituciones. Con todo, más de la mitad, valora a la democracia como la mejor forma de gobierno posible (Segob, 2013).

Se trata de tendencia que varía y que para 2016 se había incrementado. Los que más se interesan son los jóvenes de entre 25 y 34 años y los adultos de entre 45 y 54 años, así como quienes cuentan con estudios de preparatoria o bachillerato y licenciatura y posgrado, especialmente los identificados con el PRI y residentes de la zona centro-occidente del país. Los que menos interesados de mostraron fueron los mayores de 65, con estudios hasta secundaria y del centro del país. Otro dato relevante es que los apartidistas o los identificados con el partido Morena, son los menos interesados en política (Fix-Fierro et al., 2017).

Entre otras manifestaciones de cultura política, los mexicanos evidenciaron la importancia de la participación en cuanto a la organización como fuente de solución de problemas. Sin embargo, $44 \%$ de ellos encuentran muy difícil la coordinación con otros ciudadanos (Segob, 2013). Esta dimensión remite a la categoría de solución colaborativa de problemas de la cultura participativa (Jenkins, 2006).
En general, "el análisis empírico muestra que la frecuencia en la participación ciudadana para la solución de problemas comunitarios es baja y está asociada a una serie de características que están desigualmente distribuidas entre la población" (Del Tronco, 2019). Es decir, parece que no hay condiciones equitativas para la participación y tampoco hay ejercicios de integración que permitan tejer vínculos hacia la organización. Este describe una relación entre ciudadanía, pero también es resultado de la articulación que existe (o no) con las instituciones políticas.

El interés por la información entorno a asuntos políticos varía en distintos momentos, como en elecciones o en reformas jurídicas. La escolaridad, por ejemplo, se encuentra relacionada y, a nivel nacional, a mayor escolaridad, más es el interés por buscar información. Lo mismo ocurre con el ingreso en salarios mínimos (Fix-Fierro et al., 2017).

También varía el medio por el cual se busca informarse. Entre 2013 y 2016, el acercamiento a programas o noticas sobre política disminuyó, específicamente en la televisión. Como es de esperase, el uso de Internet como medio informativo, ha aumentado. Este cambio, no es homogéneo ni tampoco es tan drástico. La televisión sigue siendo, en el orden nacional, el principal medio para enterarse de asuntos diversos, incluidos los políticos. Por supuesto, esto ocurre de manera diferenciada según factores como la edad, el sexo y la escolaridad (Fix-Fierro et al., 2017).

En México, alrededor de 63 millones de habitantes tienen algún perfil en redes sociales, y es uno de los países que más tiempo pasa en redes sociales, particularmente Facebook, Instagram y Whatsapp. "Una perspectiva analítica de las elecciones recientes, tanto de México como otros países, permite concluir que el internet y las redes sociales han pasado a formar una parte relevante de la política" (Mercado, 2018).

El creciente uso de Internet como medio de información resulta alentador a primera vista. Las redes sociales abren la posibilidad a discusiones que, aparentemente, se están sosteniendo en torno a la política. Un ejemplo de ello fueron las más recientes elecciones mexicanas, en las que las redes sociales reflejaron, en buena medida, lo que sucedió. La alta participación de voto en las urnas se notó en la actividad en redes (Vázquez, 2018). 
El caso del candidato Andrés Manuel López Obrador ilustra este punto, se mantuvo como tema principal en las conversaciones, fuera con alusiones positivas o negativas. La intención de voto en las encuestas en línea lo mantuvieron a la cabeza durante la campaña y en la jornada electoral. Ambos datos apuntaban a lo que sucedió: el candidato fue vencedor y fue rápidamente reconocido por sus contrincantes (Vázquez, 2018). La situación expuesta puede entenderse como una muestra de prácticas políticas participativas, concentradas en la distribución de información (Kligler-Vilenchik and Shresthova, 2012).

A pesar de la diversidad de medios para informarse, tanto Fix-Fierro (2017) y sus colaboradores, como García (2018), han encontrado que, en general, existe un desconocimiento sobre las instituciones, ocupaciones y funcionarios. Por ejemplo, encontraron un nivel de conocimiento bajo en cuanto a las atribuciones del presidente, la ignorancia de cifras económicas y falta de información en cuanto a las cámaras del Congreso de la Unión.

Los datos anteriores sustentan la caracterización del país como uno de cultura política de desafección. Acorde al planteamiento de Thompson (1993), este trabajo se ha centrado en la producción y reproducción de formas simbólicas en la esfera pública queretana. Querétaro expresa la duración, la permanencia. Es el lugar en donde los cambios parecen transitorios para regresar siempre a lo constante. La primera se relaciona con la confianza de la ciudadanía en sus instituciones y la segunda, con la eficacia y el interés. La confianza política se integra de elementos cognitivos, evaluativos y afectivos, relacionados con la historia política tanto como con nociones circunstanciales (Almond y Verba, 2001).

La relación entre el desarrollo económico-industrial de la entidad y su historia política no es lineal, pero muestra con claridad las tensiones y articulaciones entre los grupos políticos tradicionales, herederos de los acuerdos sostenidos desde la Colonia (burguesía agrícola y comercial, etc.) y los nuevos grupos políticos (burguesía industrial, así como los grupos migrantes y los universitarios). Sin duda el México priista abrió oportunidades a la participación de estos grupos tanto para el acceso igualitario al botín del poder político, como para participar en la transformación económica, social y política.
Los equipos políticos se mantuvieron en manos de los antiguos propietarios de las haciendas, esquema que duró entre 1930 y 1970, pese a los cambios habidos en la gubernatura y otros altos funcionarios (Morales, 1998).

Mejorar la situación económica de las clases políticas y empresariales fue la divisa del desarrollo estabilizador en Querétaro, donde no hubo visos de experimentos socializantes ni movimientos populares capaces de desbaratar realmente el poder de estas élites. La "queretaneidad" (los valores autóctonos del estado, o más bien de la capital) es el término más usado por los representantes de estas clases dominantes. La construcción de una identidad oficial de la región se funda sobre un conjunto de estereotipos.

Desde 1997, Querétaro cuenta con tres alternancias electorales en 2009 y 2015 las más recientes. Este proceso inició en los municipios cercanos a la capital. Con la tercera alternancia en Querétaro se puede considerar que existe una "normalidad" electoral.

Desde que normalizó esta alternancia y con instituciones de vigilancia del voto como el IFE, hoy INE, los ciudadanos fueron capaces de expresar sus necesidades y los políticos de escucharlos, al menos en el papel. "Las elecciones, por tanto, empezaron a servir como un contrapeso a los políticos, bajo el supuesto de que los ciudadanos, al decidir por quién votar, hacen un análisis de la situación política para decidir por quién hacerlo" (Fix-Fierro et al., 2017, p. 142). Debido a este elemento analítico es relevante conocer la forma en la que los ciudadanos adquieren información para ejercer su elección. Esta búsqueda de información, por lo tanto, es una muestra más de cultura política que, a su vez se aproxima directamente a la cultura participativa que describe Jenkins (2006) en su categoría de circulación o intervención en el flujo de información.

En términos de cultura política, los cambios son poco perceptibles en el ámbito público. Históricamente la vida pública de Querétaro ha sido referida como poco participativa. Por ejemplo, son escasas las manifestaciones políticas y contadas las movilizaciones sociales. La cultura política dominante está estrechamente ligada al desarrollo económico-industrial de la entidad. La capacidad de adaptación de las élites tradicionales a los cambios de coyuntura habidos en el entorno económico y político, sobre todo a partir de los años cuarenta, tuvo como 
resultado la transformación de los hacendados en empresarios (Nieto, 2000, p. 105) y la cooptación de cuadros políticos, intelectuales y personal migrante que fueron incorporándose, en forma armónica, al poder empresarial y político de Querétaro.

De acuerdo con Diaz (2011), en los años del Querétaro posrevolucionario y la consolidación de los regímenes priistas los asuntos políticos se resolvían a nivel cupular. Esto se ha mantenido en las últimas décadas, han sido las élites las que se han traspasado los poderes, dominan los acuerdos y la vida pública se mantiene en un equilibrio que pocas veces manifiesta sobresaltos. Prueba de ello es la alternancia electoral de 1997, la primera en setenta años, en contraste con las entidades vecinas, Guanajuato y San Luis Potosí, estados que en los años previos tuvieron conflictos electorales importantes, Querétaro tuvo una alternancia imperceptible. En los años posteriores son mínimos los ejemplos de manifestación pública, los medios de comunicación han mantenido pocas tensiones con los poderes políticos $y$ económicos, y se permiten los acuerdos con las élites a fin de mantener los equilibrios (Corral, 2014).

Estas condiciones han construido la cultura política del estado. Por lo tanto, es relevante indagar sobre cómo se materializan estas circunstancias en la cultura política cotidiana de los queretanos.

\section{El análisis y levantamiento de datos}

Para el análisis de las prácticas políticas que dieran indicios para entender la cultura participativa y la cultura política, se llevó a cabo un sondeo no probabilístico con 300 cuestionarios aplicados a los habitantes de la ciudad de Querétaro, en diferentes zonas de la ciudad.

El sondeo consta de siete preguntas dirigidas a conocer las prácticas políticas y de participación de los encuestados. Las respuestas se limitaron a una por cada pregunta, según la afirmación que más se aproximara a su sentir. Las preguntas corresponden con las de la (Segob, 2013), García (2018) y Fix-Fierro, Flores y Valadés (2017). Se emplearon estos referentes para reconocer los ítems que, en general interesan en la aproximación a la cultura política. El análisis de las respuestas permite apuntar el conjunto de prácticas políticas y de participación en la esfera de lo público, teniendo como parámetro de referencia el nivel nacional. Con ello se pretende poner de manifiesto, primero, si prevalecen las prácticas arraigadas a una cultura política autoritaria y, segundo, de qué manera es posible observar esto desde los intereses que se levantan desde la cultura participativa.

Si bien se trató de un sondeo no probabilístico, el levantamiento recrea, aproximadamente las condiciones de estratos socioeconómicos de Querétaro y su zona metropolitana. Para la zona, en 2016 se detectó que 13\% de sus habitantes se encontraron en niveles $\mathrm{A} / \mathrm{B}$ es decir, alto y muy alto; $20 \%$, se podían clasificar en un nivel medio alto, $\mathrm{C}+; 19 \%$ se encontraban en un nivel medio $\mathrm{C}$; y el $15 \%$ en medio bajo, C-. Con característica de nivel bajo, en la zona metropolitana de Querétaro, se encontraba $11 \%$ de su población, D+; y $17 \%$ en muy bajo, D. Finalmente, $4 \%$ de la zona se encuentra con características de precariedad, en nivel E (AMAI, 2017).

La muestra del estudio, al realizarse en distintas colonias de la zona, presenta la misma concentración en niveles socioeconómicos medios, aunque con algunas variaciones (Figura 1). Permitiendo aproximarse a la cultura política que atraviesa este contexto en particular. Se adelanta al lector lo que se irá detallando con los resultados: la cultura política de desafección es común a todos los niveles socioeconómicos. No se encontraron relaciones significativas entre esta variable y las demás por lo que se observó un entorno se apatía generalizada.

Figura 1. Niveles socioeconómicos en la ZMQ y la muestra.

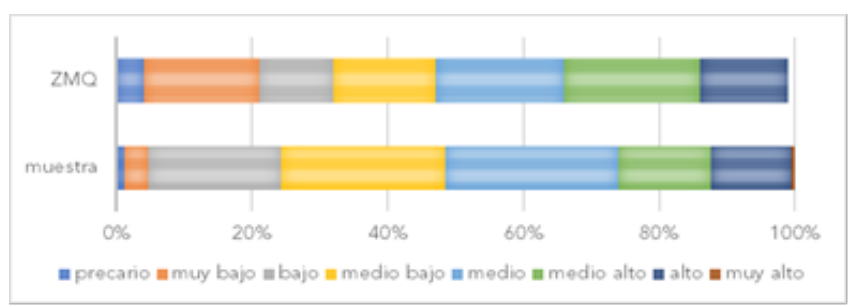

Fuente: elaboración propia para la muestra y AMAI (2017) para la ZMQ.

En el mismo sentido, los resultados describen una cultura política escasa, que parte de una opinión negativa del quehacer político y que, por lo tanto, genera muy poco interés en los ciudadanos. Destaca que los queretanos encuestados eligen enterarse de asuntos políticos por las redes sociales 
e Internet, además de sus entornos familiares. En cuanto a la participación, se encontró que hay una participación sistemática en las votaciones. Sin embargo, la práctica de reunión o agrupación en torno a intereses políticos es muy baja. A continuación, se describen los resultados específicos.

El interés de la primera pregunta se centró en conocer cuál es la opinión que se tiene sobre la política (Figura 2). Se considera un punto de partida importante puesto que las respuestas que se dieran a esta pregunta sirven como línea de análisis respecto de las siguientes preguntas.

Figura 2. Opinión acerca de la política.

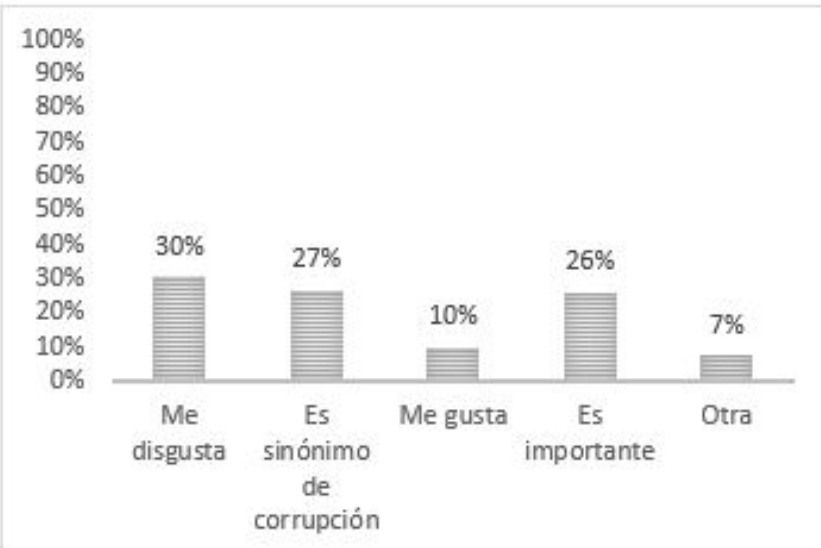

Fuente: elaboración propia.

Lo relevante de las respuestas que se muestran es que el porcentaje entre "me disgusta", "es sinónimo de corrupción" y "es importante" es similar. Ofrece una ventana para entender que, a pesar de las alternancias electorales y la competencia entre partidos políticos en los últimos veinte años, la idea sobre el ejercicio de la política no ha cambiado. Este resultado local verifica lo encontrado por Fix-Fierro, Flores y Valadez (2017) nacionalmente.

También se encontró que, a pesar de la diversificación de fuentes para obtener y compartir información, la idea que se tiene acerca de la política se reproduce. Espacios como las redes sociales, en los que se comparten opiniones en diversos formatos, incluyendo aquellos con humos como los memes, participan de la formación de opiniones, en este caso, de la persistencia de la percepción de la política como corrupción.

La opinión sobre política y el interés en ella están vinculadas. La relación se hace evidente cuando se observa la correlación entre las variables del interés y la opinión en política.

Figura 3. Interés en los asuntos políticos.

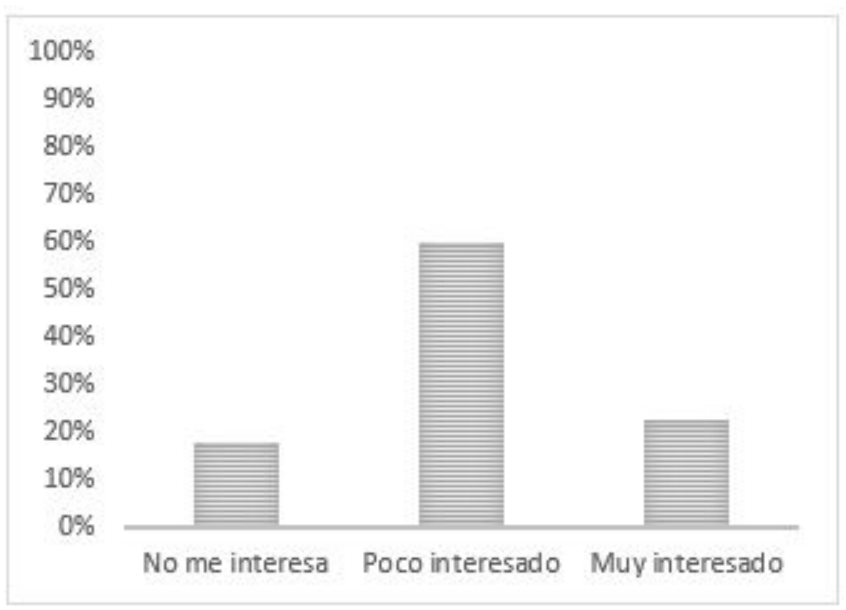

Fuente: elaboración propia.

En la Figura 4 es posible observar con detalle este vínculo. Destaca la coincidencia entre la proporción de encuestados a quienes les disgusta la política y aquellos que se muestran desinteresados.

Figura 4. Opinión e interés en política.

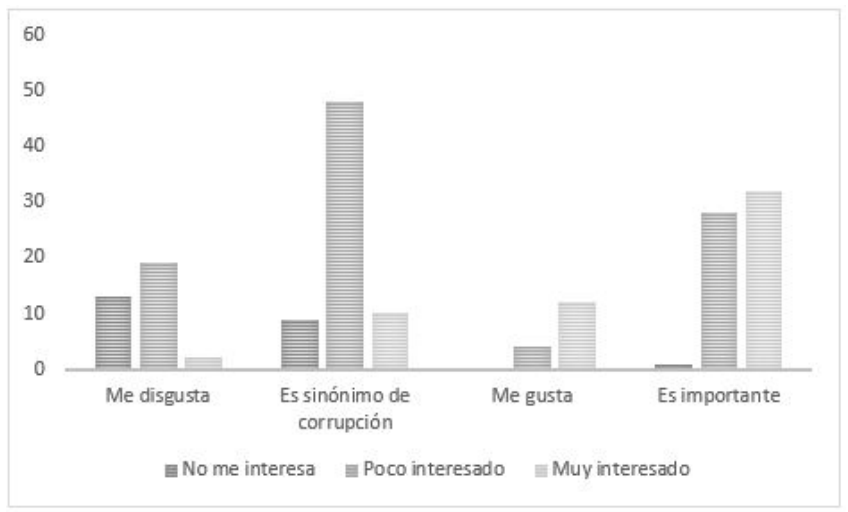

Fuente: elaboración propia.

Incluso la concentración de respuestas en "poco interesado", que se reparte en las distintas opiniones sobre política, describe un comportamiento poco comprometido con los temas políticos. Esta apreciación se reitera a través de todos los niveles socioeconómicos de la ciudad de Querétaro. Lo que predomina es la cultura de desafección que, 
aparentemente, se reproduce e integra en las conversaciones que se puedan sostener en redes sociales y otros medios participativos.

Cabe recordar que, desde la cultura participativa, la construcción y discusión de opiniones es ya un nivel de participación (Kligler Vilenchik, 2016). La formación de la opinión se construye por la mediación de las interacciones que se sostienen en línea así como fuera de ella. Por lo tanto, se observó que esta cultura y opinión atraviesan medios como estratos socioeconómicos, niveles de localidad y características sociodemográficas.

Con el propósito de particularizar la cultura participativa, en relación con la cultura política, se requería saber el nivel de interés de los sujetos cuestionados respecto a los asuntos políticos (Figura 3). El dato es contundente: casi $70 \%$ de los participantes se manifiesta poco interesado o señala que no le interesa. Relacionado con la primera pregunta, sorprende de alguna manera, puesto que la opinión sobre la política continúa siendo negativa, es casi indudable que la respuesta sea que existe poco interés en los asuntos relacionados con la vida pública.

A partir de lo anterior, correspondió conocer la opinión que tienen los encuestados de la democracia que se vive en su país (Figura 5). Queda de manifiesto que, a partir del sentido de lo que debería ser la democracia para $70 \%$ de los participantes del sondeo, México no parece serlo. Esto vincula sus propios significados contra las experiencias cotidianas. $\mathrm{Y}$ parece aún más curioso si se contrasta con la asistencia a las votaciones.

Esta variable presenta una correlación baja, casi nula con el estrato socioeconómico de los encuestados $(\mathrm{R}=0.1)$. La diferencia de percepciones atraviesa los niveles. Esto es lógico en una sociedad como la queretana o la mexicana, en la que existen diversas relaciones de dominación y desigualdad a parir de una amplia variedad de clases sociales, etnicidades, creencias y otros elementos. En un contexto como este, cada sector, cada grupo e, incluso, cada individuo construye significados distintos sobre aspectos similares. Por ejemplo, en este caso, qué es la democracia y cómo debería observarse (Tejera, 1998).

A partir del análisis se ve cómo interactúan estas dos variables (Figura 6). Se puede observar que aquellos que
Figura 5. Democracia mexicana.

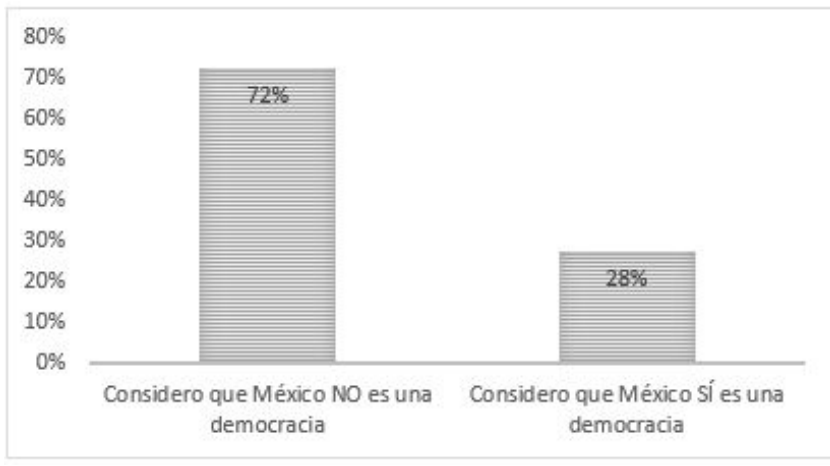

Fuente: elaboración propia.

consideran que México no es una democracia, tienden a interesarse poco o nada por la política. Algo más interesante ocurre en quienes se muestran muy interesados. En este grupo, $29 \%$ consideran que México no es una democracia y $28 \%$ opina lo contrario. Es un porcentaje similar que se interesa y permite pensar que reflexiona sobre las características de la democracia en el país, llegando a conclusiones opuestas. Esta variación de percepciones puede deberse a la complejidad del proceso de democratización por el que transita México, y la forma en la que se observa en la vida cotidiana y se representa en los medios.

Figura 6. Opinión sobre la democracia mexicana e interés en política.

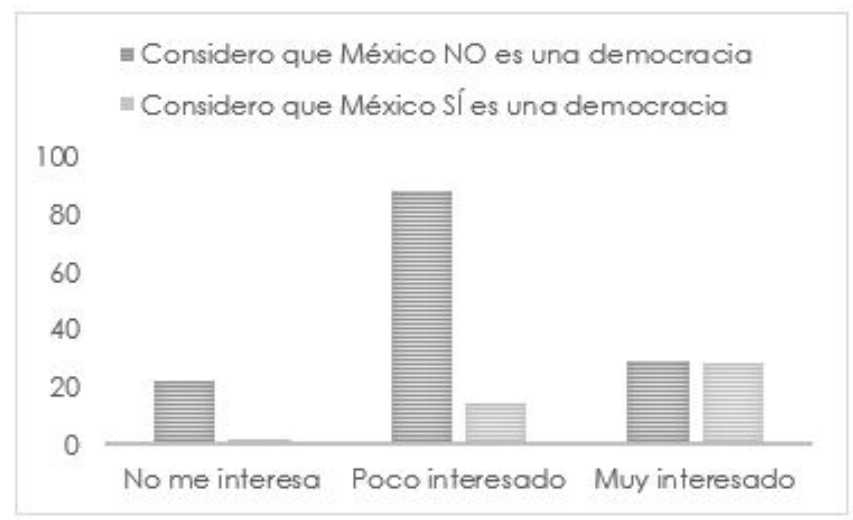

Fuente: elaboración propia.

A pesar de los resultados anteriores, se encontró una alta participación en las mesas electorales como un ejercicio primigenio de participación política (Figura 7). Más aún, la 
población entrevistada pensaba votar el próximo año, en las elecciones de distintos órdenes de gobierno que ocurrieron en julio de 2018 (Figura 8). La idea de participación y de cultura política se circunscribe a las prácticas electorales. Sin importar la opinión que se tenga sobre la política o sobre los asuntos políticos, la población considera acudir a las urnas como parte de un ejercicio democrático y ciudadano.

La votación se describe como la forma de participación democrática más representativa y sintomática. Esto es por tres razones. Primero, porque es la forma de expresión democrática a la que se tiene un acceso más o menos igualitario. En segundo lugar, permite una vinculación directa entre el electorado y el poder que se manifiesta en su elección de representantes. Finalmente, porque los resultados de las elecciones tienen repercusiones directas en las políticas públicas y sus consecuencias sociales, políticas y económicas (Nohlen, 2004).

La traducción de este interés por el momento de la votación se evidenció en la actividad en redes sociales durante las campañas previas a la elección y específicamente en jornada electoral (Mercado, 2018 y Vázquez, 2018). Las conversaciones, las discusiones, hasthags y memes se concentraron en el asunto de candidatos y el voto.

Figura 7. Participación en las elecciones.

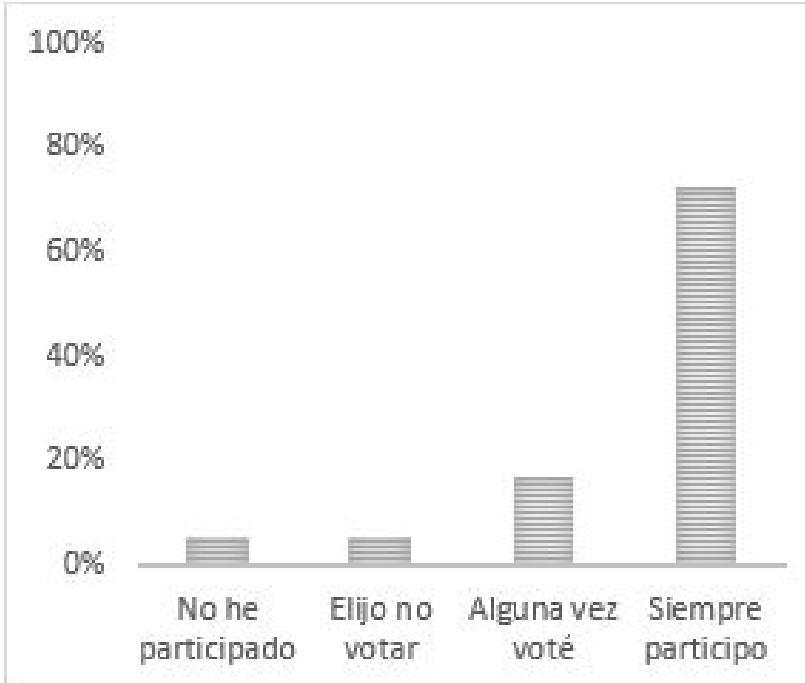

Fuente: elaboración propia.
Estos resultados (Figura 7 y Figura 8) evidencian que existe una normalidad electoral, es decir, la población acude a las urnas de manera habitual. Se reconoce como parte de las rutinas políticas los procesos electorales, como un ejercicio básico de prácticas políticas. Nohlan (2004) lo explica en el campo de lo simbólico de lo político. En este entorno y en el contexto de Querétaro, determinados fenómenos, especialmente el de participar en las votaciones, se carga de normalidad y emotividad. Es decir, se le asocia como algo positivo y la sociedad exige a sus integrantes, en las interacciones cotidianas, cumplir con el derecho y la obligación de votar. Así se refuerza el ejercicio y legitima el modelo de democracia, en línea así como fuera de ella, con los distintos matices que ofrecen la variedad de interacciones.

Figura 8. Considera votar en 2018.

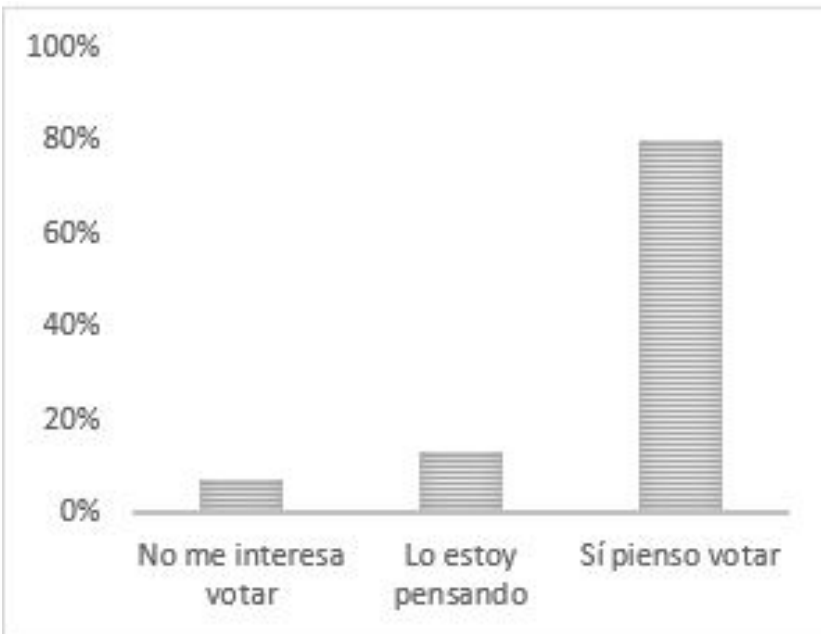

Fuente: elaboración propia.

En este contexto, el ejercicio de la cultura política se ejerce cada vez más en espacios simbólicos, aunque sin abandonar los espacios tradicionales de ejercicio político. No es un fenómeno espontáneo. Desde finales de la década de 1970 y durante aquella de 1980, han venido emergiendo nuevos actores con nuevas formas de expresión política, no necesariamente vinculadas a la institucionalización de la política. Cada una con sus reivindicaciones y luchas, con manifestaciones propias que han tenido que buscar o crear espacios de diálogo. Esto no necesariamente ha significado la construcción de una comunicación eficiente o un proceso de democratización efectivo. Al contrario, parece ser que, 
simplemente, se han diversificado los espacios para llevar a cabo prácticas añejas (Pineda, 2010).

Vale la pena diferenciar entre participación política y participación electoral. La participación política incluye a la participación convencional, articulada a las instituciones, como la pertenencia a grupos representativos. Sin embargo, el concepto y las actividades se han extendido para abarcar formas emergentes, por fuera de las instituciones políticas establecidas (Nohlen, 2004).

Entre las prácticas de cultura política se encuentra la recopilación de información de este tipo (Figura 9). Esa forma de participación es menos accesible para la generalidad de la población. Requiere una inversión de tiempo, recursos materiales e intelectuales. Es necesaria la capacitación en la búsqueda, filtrado, adquisición y comparación de información relevante y pertinente. Por esta razón, interesó conocer el medio por el que las personas conocen de política, pensando en que las decisiones y actitudes con respecto a la política se construyen a partir de datos y opiniones recolectadas provenientes de distintos medios. Además, uno de los debates sobre cultura participativa radica en el uso de los medios y el consumo de estos. Queda claro que las redes sociales virtuales, la televisión y la Internet son los espacios a través de los cuales se va construyendo el debate sobre los asuntos políticos.

Los medios masivos clásicos se mantuvieron, por mucho tiempo, como fuentes de información política importantes, aunque unilaterales, para los ciudadanos (Pineda, 2010). $\mathrm{Su}$ relevancia, sin duda, se mantiene. De acuerdo con lo recuperado, $42 \%$ de los encuestados mantiene como su principal fuente de información a la televisión, la radio o el periódico.

En la actualidad, gracias a la presencia de las tecnologías de la información y la comunicación, el rol del suministro de información es más diverso. A través de la Internet es posible construir alternativas de información, comunicación y participación política más dinámicas y bilaterales (Pineda, 2010).

Esta variable se asocia con un primer nivel de prácticas cívicas o políticas, aquellas que se concentran en la adquisición y, en su caso, la distribución de información. Apunta a aprender algo de los temas sociales. También significa que los individuos sean capaces de informar a otros causas, procesos e implicaciones (Kligler-Vilenchik and Shresthova, 2012).

Figura 9. Medio para enterarse de política.

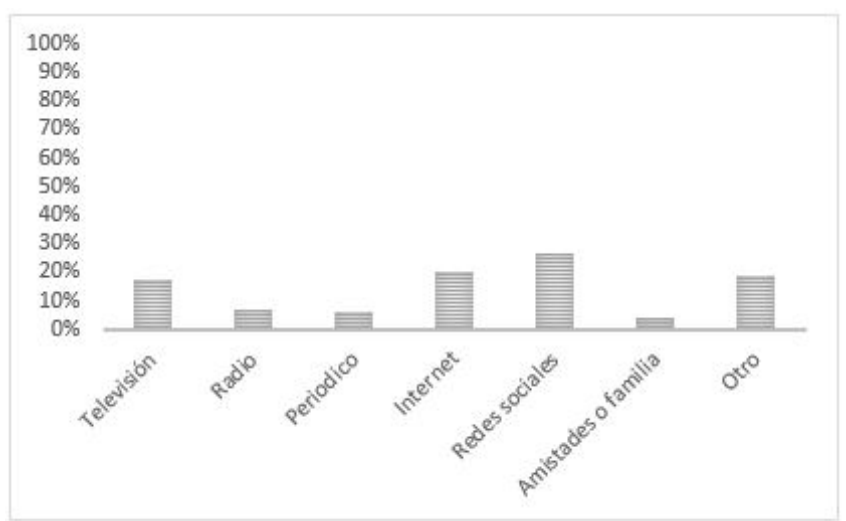

Fuente: elaboración propia.

Este dato invita a pensar sobre las construcciones expresivas que se producen dentro de la cultura particular de la ciudadanía queretana. La información que se genera en estas redes parece estar atrayendo el interés de los usuarios, al mismo tiempo, está conquistando espacios en donde debatir lo político a partir de la adquisición de habilidades de la cultura participativa. Es preciso, por lo tanto, conocer el uso que se le da a esta información y a los medios, sobre todo, los digitales.

Al mismo tiempo es interesante pensar en los resultados junto con la idea de democracia que se tiene de México. En todo caso, los significados sobre la democracia y, en general, lo político y lo público se construyen a partir de las interacciones que se tiene con otros. El hecho de que estas ocurran por vía de los medios de comunicación y en menor medida con el entorno directo, invita a reflexionar sobre las propuestas de significados que se ofrecen por los medios.

Desde la concepción liberal de la participación política, la variabilidad en la participación y en la intensidad de la misma, se habilitan las dinámicas y la racionalidad de los procesos políticos democráticos. Con el acceso abierto a los procesos, la comunicación dirigida a promover la participación funciona, al mismo tiempo, como incentivo del propio modelo político (Nohlen, 2004).

La presencia predominante de las redes sociales en la comunicación de temas políticos describe nuevas interacciones en nuevos espacios de información y 
comunicación. La tecnología de estos, media prácticas de ciudadanía emergentes que buscan alternativas de discusión y de visibilización, es decir, de poder (Pineda, 2010).

Sin embargo, no es posible afirmar que simplemente por el uso de nuevas tecnologías que suministran información o la diversidad de las fuentes, o se esté construyendo una cultura política más interesada o participativa. Es decir, no se puede afirmar, a partir de los datos recabados, que las redes o, en general, el entorno de Internet sea necesariamente aprovechado para construir nuevas prácticas. Al contrastarlo con el resto de las respuestas, es más bien posible cuestionar si la relación con los medios digitales reproduce la que ya se tenía con los tradicionales. Si bien existe la posibilidad de generación de nuevas prácticas, también es posible que su operación imite la unidireccionalidad y la escasez de participación.

Esta última idea puede reforzaste con el ejercicio de otra práctica de cultura política, que es la participación directa en comunidades y organizaciones políticas o de representación (Figura 9). Si bien se trata de participación vinculada a la institucionalidad, su activación describiría cierto interés en los asuntos de la política. Lo cual no sucede. A pesar de la constante participación en las elecciones, la población se mantiene alejada de las organizaciones políticas, ni propias ni creadas por alguien más.

Figura 10. Participación en alguna organización política o de representación

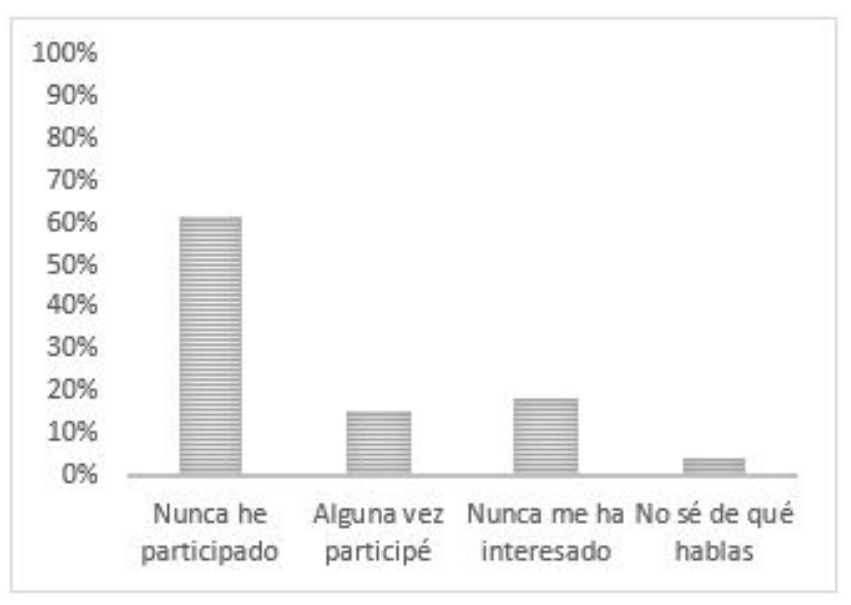

Fuente: elaboración propia.
Este dato es relevante desde la cultura política así como desde la participativa. Implica, por un lado, que no hay un interés por participar en política. Se describe que se participa poco de la vida pública con el argumento de que la política es para los políticos. Por otro lado, las destrezas aprendidas para conseguir y difundir información tampoco trascienden hacia la categoría activa propia de la cultura participativa.

Para ahondar en esta área, se apunta a pensar cómo se discute o referencia la política en entornos ajenos a ellos pero que atraen la participación, como grupos religiosos, deportivos o de algún interés de consumo en específico. Interesará ver cómo se aprovechan las redes sociales, virtuales o físicas para deliberar asuntos de interés común, así como a percepción de la democracia o de sus representaciones y representantes.

\section{A manera de cierre}

Al observar los resultados en el contexto de Querétaro, se vio que los informantes manifiestan la misma desafección y alejamiento por los asuntos públicos que se ha encontrado a nivel nacional. Esto, a pocos años de la alternancia democrática y al contrario del entusiasmo que se consideraría encontrar en las primeras fases del proceso de democratización. Comparados con los conceptos, los medios no cumplen con su parte de erigirse en vínculo crítico entre sociedad civil y sociedad política. Aun teniendo claro que son actores políticos, continúan manteniendo los vínculos con los actores dominantes.

Lo anterior remite a una cultura política autoritaria que mantiene la continuidad histórica, conservadora y vertical del estado. Si bien Querétaro es una de las entidades que más participa en los procesos electorales, cuando se trata de involucrarse en asuntos que trasciendan lo electoral, la participación pasa a ser mínima y estrictamente coyuntural.

Los resultados del estudio demuestran la necesidad de ir más allá de los procesos normativos en donde regularmente se emplea. Se visualizó que vale la pena contemplar con mayor profundidad las actividades políticas que aprovechan entornos digitales y, de esta manera, solidificar el análisis entre la cultura política y la cultura participativa. Interesa entender si la cultura política se ha transformado. Hay quien hipotetiza que la participación política, a través de las redes virtuales, origina una participación multicultural en la que tienen lugar debates 
y prácticas entre distintos grupos de interés. Incluso se les sueña como espacios de organización que aglutinan a sujetos de distintas culturas con intereses comunes que trascienden los tradicionales de la política (Pineda, 2010).

Los resultados exploratorios son menos optimistas. Se encontró que son espacios de diversificación de la oferta simbólica en los que conviven intensiones y prácticas emergentes, con anclajes más viejos. Asu vez, se descubrió que funcionan como adaptaciones de procesos con raíces muy antiguas. Esto se sostiene en Querétaro como en Latinoamérica. Se perpetúan este tipo de prácticas debido a:

Las condiciones socioeconómicas en los países de la región, el nivel de educación, la heterogeneidad social y cultural de las sociedades, las tradiciones y creencias, el concepto de poder y la actitud frente al mismo, el hábito de consenso - conflicto, entre otras realidades. (Nohlen, 2004, p. 152)

El proceso de transformación de Querétaro ha traído cambios tangibles en el plano institucional, cambios que han supuesto modificaciones en las organizaciones burocráticas y en la manera de construir relaciones políticas. Algunas de ellas ya se han mostrado en los resultados obtenidos; sin embargo, en las prácticas cotidianas, esta reorganización institucional apenas es perceptible, pues los discursos políticos y las representaciones en torno a la acción política parecen desvanecerse bajo el peso muerto de la cultura política consuetudinaria, basada en el control, la cooptación o la represión de todos aquellos factores que afecten el dogma de la paz social. Las representaciones y los discursos varían, pero las prácticas parecen tender a la reproducción.

Las plataformas de redes sociales se consideran como avenidas de expresión y conversación política viable y confiable, a pesar de las posibilidades de fake news. Ofrecen la posibilidad de participar de la difusión y amplificación de mensajes. Incluso son entornos de potencial persuasión de audiencias, cuidadosamente seleccionadas y a las cuales se les presentan formatos y contenidos específicos (Mor et al., 2015). Las redes sociales son mediaciones en las interacciones y, más particularmente, en la participación.
También se vio una diversificación en los medios, en la manera de interactuar con la información sobre política, tanto localmente, en este estudio, como nacionalmente (García, 2018 y Fix-Fierro et al., 2017). Se evidencia a las redes sociales como espacios de obtención de información, por lo que se intuye una participación en la divulgación de esta, sino de todos los encuestados, si de ciertos elementos en sus redes que se integran al flujo de información. Sin embargo, lo que se distingue de estas interacciones es una reproducción de percepciones y opiniones compartidas previamente.

Habermas (1981) recuerda siempre y en todas partes el deber ser de la participación pública como referente empírico para la sana configuración de la esfera pública. Las evidencias que surgen de este estudio revelan cuán lejos está Querétaro de la participación y la deliberación racional en asuntos de interés público. Este escenario se revela como ideal para analizar las contradicciones que se dan entre un entorno institucional democratizado y un entorno marcado por la cultura política tradicional.

Es necesario insistir en este estudio como un primer acercamiento que dejó más cuestionamientos que certidumbres. Será necesario ampliarlo en cuento a alcance, pero sobre todo seguir desdoblando el interés en la cultura política hacia prácticas más recientes de cultura participativa. Puntos como los participantes, las agrupaciones, los intereses compartidos, las formas de discusión, los formatos que adquiere la información que se comparte y las reacciones hacia ellas, podrían enriquecer este primer interés.

Sin embargo, destaca el potencial explicativo del tejido conceptual de cultura política y cultura participativa. Apunta a que puede servir para dar cuenta de cómo interactúan las nuevas y las viejas formas de ejercicio del poder, tanto institucional como alternativo. Puede así dar cuenta de elementos que inciden en distintos fenómenos de comportamiento político, desde la votación hacia distintos y tal vez nuevos constructos de activación política. Su potencia recae en que se fundamenta en el aspecto cultural de la participación. En este sentido, permite examinar cómo “en la cultura política hay más cultura que política" (Tejera, 1998, p. 150). 


\section{Referencias}

Almond, G., Verba, S., 2001. La cultura política, in: Battle, A. (Ed.), Diez Textos Básicos de Ciencia Política. Ariel, Madrid, pp. 171-201.

AMAI, 2017. Delimitación de zonas metropolitanas, Niveles Socioeconómicos. Asociación Mexicana de Agencias de Investigación de Mercado, Ciudad de México.

Andrade, P., 2009. La democracia en el espacio público mediático. Glob. Media J. 6, 91-100.

Barba, R., 2017. Otaku mexicanos. Al análisis de la cultura participativa de aficionados a las narrativas transmediales japonesas (Doctorado en Estudios Socioculturales). Universidad Autónoma de Aguascalientes, Aguascalientes, México.

Corral, G.A., 2014. La configuración de la esfera pública de Querétaro. El caso de Acueducto II. (Doctorado en Estudios Científico Sociales), Instituto Tecnológico y de Estudios Superiores de Occidente, Guadalajara, México

Del Tronco Paganelli, J., 2019, febrero 8. La democracia participativa en México. ¿Hay condiciones para ejercerla? Recuperado de Oraculus website: https://or aculus.mx/2019/02/08/la-democracia-participativa-enmexico-hay-condiciones-para-ejercerla/

Díaz, A., 2002. Democratización y Alternancia. Encuesta de actitudes y orientaciones políticas de los ciudadanos en Querétaro. UAQ, UDG, IEQ, Querétaro.

Diaz, A., 2011. La paz y sus sombras. Cultura política en el Estado de Querétaro. UAQ/Miguel Ángel Porrúa, México

Fix-Fierro, H., Flores, J.I. y Valadés, D., 2017. Los mexicanos y su Constitución. Tercera Encuesta Nacional de Cultura Constitucional. Universidad Nacional Autónoma de México, México. Recuperado de http://www.losmex icanos.unam.mx/MexicanosConstitucion/pdf/Mexican os_Constitucion.pdf

García Reyes, C.U., 2018. Desafección política en México. ¿Es factible incrementar el interés de los mexicanos en la política? Visor Ciudadano (62). Instituto Belisario Domínguez, Senado de la República.

Habermas, J., 1981. Historia y crítica de la opinión pública. La transformación estructural de la vida pública. Gustavo Gili, México.

Jenkins, H., 2006. Fans, bloggers, and gamers: exploring participatory culture. New York University Press, New York.

Jenkins, H., 2008. Convergence culture: la cultura de la convergencia de los medios de comunicación. Paidós, Barcelona.

Jenkins, H., 2009. Confronting the challenges of participatory culture: media education for the 21st century, The John D. and Catherine T. MacArthur Foundation Reports on
Digital Media and Learning. The MIT Press, Cambridge, MA.

Jenkins, H., John, N., \& Kligler-Vilenchik, N. (2019, 26). Participatory Politics in an Age of Global Crisis: Neta Kligler-Vilenchik and Nicholas John (Part Two),, de Henry Jenkins: Confessions of an Aca Fan website: http: //henryjenkins.org/blog/2019/3/10/participatory-politi cs-in-an-age-of-global-crisis-neta-kligler-vilenchik-a nd-nicholas-john-part-two

Kligler-Vilenchik, N., Shresthova, S., 2012. Learning Through Practice: Participatory Culture Civics (Reporte de estudio de caso), Media, Activism and Participatory Politics Project. Civil Paths. Annenberg School for Communication and Journalism. Univesity of Southern California.

Kligler-Vilenchik, N. (2016). Mechanisms of translation: From online participatory cultures to particpatory politics. Journal of Digital and Media Literacy, 4(1-2).

Larabie, C., 2011. Participatory Culture and the Hidden Costs of Sharing. McMaster J. Commun. 7, 4.

Mattelart, A., 2003. Geopolítica de la cultura. Ediciones desde abajo, Bogotá, Colombia.

Mercado Ramírez, M. L. (2018, octubre 1). La influencia de las redes sociales en la política., de Foro Jurídico website: https://forojuridico.mx/la-influencia-de-las-r edes-sociales-en-la-politica/

Mor, Y., Kligler-Vilenchik, N., \& Maoz, I. (2015). Political Expression on Facebook in a Context of Conflict: Dilemmas and Coping Strategies of Jewish-Israeli Youth. Social Media + Society, 1(2), 205630511560675. https: //doi.org/10.1177/2056305115606750

Morales, M., 1998. La nueva generación de políticos queretanos. UAQ, Querétaro.

Nohlen, D., 2004. La participación electoral como objeto de estudio, in: Reyna, C. (Ed.), Elecciones. Oficina Nacional de Procesos Electorales (ONPE), Lima, Perú, pp. 137-158.

Pineda, M., 2010. Las nuevas prácticas ciudadanas en internet y las oportunidades para políticas de comunicación participativas. Rev. Estud. Cult. 6, 31-46.

Scolari, C.A., 2008.Hipermediaciones: elementos para una teoría de la comunicación digital interactiva. Gedisa, Barcelona.

Segob, 2013. Encuesta Nacional sobre Cultura Política y Prácticas Ciudadanas. Disponible en línea: http://www. encup.gob.mx [29/05/13]

Tejera, H., 1998. Cultura, poder y racionalidad. Alteridades 8 , 145-157.

Thompson, J.B., 1998. Los media y la modernidad: una teoría de los medios de comunicación. Paidós, Barcelona.

Thompson, J.B., 1993. Ideología y cultura moderna. Teoría crítica social en la era de la comunicación de masas, 2a ed. Universidad Autónoma Metropolitana, 
Unidad Xochimilco, División de Ciencias Sociales y Humanidades, México, D.F.

Thorburn, D., Jenkins, H., Seawell, B., 2003. Rethinking media change: the aesthetics of transition. MIT Press, Cambridge, Mass.

Vázquez, R. (2018, julio 2). Las redes sociales durante la jornada electoral 2018, de Forbes México website: https: //www.forbes.com.mx/las-redes-sociales-durante-la-jo rnada-electoral/ 